\title{
A Reliability Sensitivity Analysis Method Based On Saddlepoint Approximation
}

\author{
Hao Lu \\ College of Mechanical and Electrical Engineering, China University of Mining and Technology, Xuzhou, China
}

Keywords: Structural reliability, Parameter sensitivities, Moment, Saddlepoint approximation, Statistical perturbation.

\begin{abstract}
Reliability sensitivity analysis quantifies the effect of input random variables on model outputs. An efficient reliability sensitivity analysis method is developed herein by combining the stochastic perturbation technique and a third-moment saddlepoint approximation. The presented method requires only the first three moment of the structural response and the cumulant generating function of input random variables is not strictly required. Numerical examples are illustrated and the procedure shows that the method is convenient to be applied to parameter sensitivity analysis of structural reliability.
\end{abstract}

\section{Introduction}

Uncertainty is inherent and unavoidable in almost all mechanical systems. As an important tool for quantifying and dealing with all kinds of uncertainties, reliability sensitivity analysis could provide not only the reliability level, but also the impact of the input uncertainties on the system performance. Generally, the reliability sensitivity analysis methods may be divided into sampling simulation methods ${ }^{[6,8]}$ and approximate methods ${ }^{[3,9,10,12]}$. Approximate methods such as the well-known first- and second-order reliability methods $(\mathrm{FORM} / \mathrm{SORM})^{[7]}$ and moment matching methods ${ }^{[12]}$ are often used for the failure probability evaluation. The FORM is computationally efficient, but may not be accurate for nonlinear limit-state functions due to a nonlinear non-normal to normal transformation. In order to ease the computational difficulties, moment matching methods are usually employed. This type of methods approximates the distribution of the system performance by selecting a distribution from a class of assumed distributions through fitting the first few moments of the response. The sampling simulation methods could provide more accurate results, but it is time-consuming for explicit limit state function problems, especially when the failure probability is smaller than 1e-4.

As a recently developed approximation method, the thirdmoment saddlepoint approximation (TMSA) is used which enables the description of a wide range of distribution functions based on the first few stochastic moments. The uncertainty in the required moments is estimated based on a statistical perturbation method ${ }^{[7]}$. Based on the TMSA, an efficient method for reliability sensitivity analysis considering uncertain stochastic parameters is presented. Based on approximation strategies, this extended analysis requires no additional limit state function evaluations, which are very efficient for the reliability analysis of complex models with the most time consuming part.

The paper is organized as follows. In Section 2, the statistical perturbation of the statistical response is performed. The third moment saddlepoint approximation method is discussed in section 3 . In section 4 , the reliability sensitivity approach is developed with the matrix differential technology. Two illustrative examples are used to demonstrate and examine the presented methods in Section 5. Conclusions are given in Section 6

\section{Analysis of statistical response}

A limit state function is usually defined by the performance or the failure mode of a structure in the classical reliability problem, which entails the evaluation of the multiple integral

$$
P_{f}=\operatorname{Pr}[G(\boldsymbol{X}) \leq 0]=\int_{G(\boldsymbol{X}) \leq 0} \cdots \int f_{\boldsymbol{X}}(\boldsymbol{X}) d \boldsymbol{X}
$$

where $P_{f}$ is the probability of failure, $\boldsymbol{X}_{i}, i=1,2, \ldots, n$ denotes $n$ dependent random variables with joint density $f_{X}(\boldsymbol{X})$, and $G(\boldsymbol{X})$ is a limit state function that is formulated such that $G(\boldsymbol{X}) \leq 0$ implies failure. Since $G(\boldsymbol{X})$ is usually a nonlinear function of random variables, it is usually a difficult task to find an analytical expression to the multidimensional integration. Due to the difficulty and complexity of the analytical integration of equation (1), the moment-based approaches are often selected as an alternative.

Herein, the statistical perturbation method is adopted to estimate the first few moment of the statistical response. According to the perturbation theory, the vector of random variable $\boldsymbol{X}$ and the limit state function $G(\boldsymbol{X})$ could be expanded as

$$
\begin{gathered}
\boldsymbol{X}=\boldsymbol{X}_{\mathrm{d}}+\varepsilon \boldsymbol{X}_{\mathrm{p}} \\
G(\boldsymbol{X})=G_{\mathrm{d}}(\boldsymbol{X})+\varepsilon G_{\mathrm{p}}(\boldsymbol{X})
\end{gathered}
$$

where $\varepsilon$ is a small parameter. The subscript $\mathrm{d}$ and p represents the certain part and the random part of the random parameters, respectively. The random parameters have a zero mean value. The value of the random part should be smaller than the value of the certain part. Both sides of equations (2) and (3) are evaluated with respect to the mean of random variables as

$$
\mathrm{E}(\boldsymbol{X})=\mathrm{E}\left(\boldsymbol{X}_{\mathrm{d}}\right)+\varepsilon \mathrm{E}\left(\boldsymbol{X}_{\mathrm{p}}\right)=\boldsymbol{X}_{\mathrm{d}}=\overline{\boldsymbol{X}}
$$




$$
\begin{aligned}
\mathrm{E}\lfloor G(\boldsymbol{X})\rfloor & =\mathrm{E}\left\lfloor G_{\mathrm{d}}(\boldsymbol{X})\right\rfloor+\varepsilon \mathrm{E}\left\lfloor G_{\mathrm{p}}(\boldsymbol{X})\right\rfloor \\
& =G_{\mathrm{d}}(\boldsymbol{X})=\bar{G}(\boldsymbol{X})
\end{aligned}
$$

Similarly, according to the Kronecker algebra, both sides of equations (2) and (3) are evaluated about the variance, the third moment of the random variables as

$$
\begin{aligned}
& \operatorname{Var}(\boldsymbol{X})=\mathrm{E}\left\{[\boldsymbol{X}-\mathrm{E}(\boldsymbol{X})]^{[2]}\right\}=\varepsilon^{2} \mathrm{E}\left\{\boldsymbol{X}_{\mathrm{p}}^{[2]}\right\} \\
& \begin{aligned}
C_{3}(\boldsymbol{X})=\mathrm{E}\left\{[\boldsymbol{X}-\mathrm{E}(\boldsymbol{X})]^{[3]}\right\}=\varepsilon^{3} \mathrm{E}\left\{\boldsymbol{X}_{\mathrm{p}}^{[3]}\right\} \\
\operatorname{Var}[G(\boldsymbol{X})]=\mathrm{E}\left\{[G(\boldsymbol{X})-\mathrm{E}(G(\boldsymbol{X}))]^{[2]}\right\} \\
=\varepsilon^{2} \mathrm{E}\left\{\left[G_{\mathrm{p}}(\boldsymbol{X})\right]^{[2]}\right\} \\
\mathrm{C}_{3}[G(\boldsymbol{X})]=\mathrm{E}\left\{[G(\boldsymbol{X})-\mathrm{E}(G(\boldsymbol{X}))]^{[3]}\right\} \\
=\varepsilon^{3} \mathrm{E}\left\{\left[G_{\mathrm{p}}(\boldsymbol{X})\right]^{[3]}\right\}
\end{aligned}
\end{aligned}
$$

where the Kronecker power is $\boldsymbol{P}^{[k]}=\boldsymbol{P} \otimes \boldsymbol{P} \otimes \cdots \otimes \boldsymbol{P}$, and the symbol $\otimes$ represents Kronecker product which is defined as $(\boldsymbol{A})_{p \times q} \otimes(\boldsymbol{B})_{s \times l}=\left[a_{i j} \boldsymbol{B}\right]_{p s \times q t} . \operatorname{Var}(\boldsymbol{X})$ and $\boldsymbol{C}_{3}(\boldsymbol{X})$ is the variance matrix and the third central moments matrix of $\boldsymbol{X}$, respectively.

By expanding the limit state function $G_{\mathrm{p}}(\boldsymbol{X})$ to the first-order Taylor series of vector-valued functions and matrix-valued functions at a point $\mathrm{E}(\boldsymbol{X})=\boldsymbol{X}_{\mathrm{d}}$, which is on the failure surface $G_{\mathrm{p}}\left(\boldsymbol{X}_{\mathrm{d}}\right)=0$, the expression of $G_{\mathrm{p}}(\boldsymbol{X})$ is given as

$$
G_{\mathrm{p}}(\boldsymbol{X})=\frac{\partial G_{\mathrm{d}}(\boldsymbol{X})}{\partial \boldsymbol{X}^{\mathrm{T}}} \boldsymbol{X}_{\mathrm{p}}
$$

Substituting equation (10) into equations (8) and (9), we obtain

$$
\begin{aligned}
\sigma_{\mathrm{G}}^{2} & =\operatorname{Var}[G(\boldsymbol{X})]=\varepsilon^{2} \mathrm{E}\left\{\left(\frac{\partial G_{d}(\boldsymbol{X})}{\partial(\boldsymbol{X})^{\mathrm{T}}}\right)^{[2]} \boldsymbol{X}_{p}^{[2]}\right\} \\
& =\left[\frac{\partial G_{d}(\boldsymbol{X})}{\partial(\boldsymbol{X})^{\mathrm{T}}}\right]^{[2]} \operatorname{Var}(\boldsymbol{X}) \\
\gamma_{G} & =\mathrm{C}_{3}[G(\boldsymbol{X})]=\varepsilon^{3} \mathrm{E}\left\{\left(\frac{\partial G_{d}(\boldsymbol{X})}{\partial(\boldsymbol{X})^{\mathrm{T}}}\right)^{[3]} \boldsymbol{X}_{p}^{[3]}\right\} \\
& =\left[\frac{\partial G_{d}(\boldsymbol{X})}{\partial(\boldsymbol{X})^{\mathrm{T}}}\right]^{[3]} C_{3}(\boldsymbol{X})
\end{aligned}
$$

where $\sigma_{G}^{2}$ and $\gamma_{G}$ are the variance and the third central moments of $G(\boldsymbol{X})$ respectively.

\section{Moment-based estimation of failure probability}

According to the moment matching method, the failure probability of a structure could be calculated by using the statistical moments of limit-state function $Z=G(\boldsymbol{X})$ and fitting the moments with some empirical distribution ${ }^{[11]}$. In order to incorporate these moments in a flexible distribution function, the third-moment saddlepoint approximation (TMSA) is adopted in the presented paper. According to TMSA proposed by pef. [4], the failure probability of a structure with the limitstate function $Z=G(\boldsymbol{X})$ can be represented as

$$
\begin{aligned}
P_{f} & =P(Z \leq 0)=P\left(Z_{s} \leq-\beta_{2}\right) \\
& =\Phi\left[r+\frac{1}{r} \ln \left(\frac{q}{r}\right)\right]
\end{aligned}
$$

in which,

$$
\begin{gathered}
Z_{s}=\left(Z-\mu_{G}\right) / \sigma_{G} \\
\beta_{2}=\frac{\mu_{G}}{\sigma_{G}} \\
r=\operatorname{sign}(\hat{\theta})\left\{2\left[\hat{\theta}\left(-\beta_{2}\right)-K_{Z s}(\hat{\theta})\right]\right\}^{1 / 2} \\
q=\hat{\theta}\left[K_{Z s}^{\prime \prime}(\hat{\theta})\right]^{1 / 2}
\end{gathered}
$$

and $\operatorname{sign}(\cdot)$ is the $\operatorname{sign}$ function with $\operatorname{sign}(\theta)=1,-1$, or 0 corresponding to the cases $\theta>0, \theta<0$, or $\theta=0$, respectively. The function $K_{\mathrm{Zs}}(\hat{\theta})$ and $K_{\mathrm{Zs}}^{\prime \prime}(\hat{\theta})$ can be expressed respectively as follows

$$
\begin{gathered}
K_{\text {Zs }}(\hat{\theta})=\left\{\begin{array}{l}
\left.-\frac{2}{\gamma_{G}} \hat{\theta}-\frac{2}{\gamma_{G}^{2}} \ln \mid\left(1-\frac{\gamma_{G}}{2} \hat{\theta}\right)^{2}\right],\left(\gamma_{G} \neq 0\right) \\
0.5 \hat{\theta}^{2},\left(\gamma_{G}=0\right)
\end{array}\right. \\
K_{Z s}^{\prime \prime}(\hat{\theta})=\frac{1}{\left(1-0.5 \gamma_{G} \hat{\theta}\right)^{2}}
\end{gathered}
$$

where $K_{Z s}$ is the cumulant generating function (CGF) of the standardized variable $Z_{s}$.

The saddlepoint equation is represented as

$$
\left(2+\gamma_{G} z\right) \theta-2 z=0
$$

While $z=-\beta_{2}$, the single saddlepoint is provided as follows

$$
\hat{\theta}=\frac{2 \beta_{2}}{\gamma_{G} \beta_{2}-2}
$$

According to the TMSA, the simplicity of the moment matching method and the accuracy of saddlepoint approximation were integrated and utilized effectively. Instead of CGFs of basic variables, only the moments of the variables is required in the TMSA.

\section{Reliability sensitivity analysis based on TMSA}

The method for reliability-based sensitivity analysis is constructed on TMSA in this section. The reliability sensitivity refers as the partial differential of the failure probability $P_{f}$ with respect to the distribution parameters (ie. the mean and the variance ) of random variables ${ }^{[2]}$. By using matrix differential technology, the reliability sensitivity with respect to the mean and the variance could be obtained as

$$
\frac{\partial P_{f}}{\partial \overline{\boldsymbol{X}}^{\mathrm{T}}}=\left(\frac{\partial P_{f}}{\partial \theta} \frac{\partial \theta}{\partial \beta_{2}}+\frac{\partial P_{f}}{\partial \beta_{2}}\right) \frac{\partial \beta_{2}}{\partial \mu_{G}} \frac{\partial \mu_{G}}{\partial \overline{\boldsymbol{X}}^{\mathrm{T}}}
$$




$$
\frac{\partial P_{f}}{\partial \operatorname{Var}(\boldsymbol{X})}=\left(\frac{\partial P_{f}}{\partial \theta} \frac{\partial \theta}{\partial \beta_{2}}+\frac{\partial P_{f}}{\partial \beta_{2}}\right) \frac{\partial \beta_{2}}{\partial \sigma_{G}} \frac{\partial \sigma_{G}}{\partial \operatorname{Var}(\boldsymbol{X})}
$$

where,

$$
\begin{aligned}
& \frac{\partial P_{f}}{\partial \theta}=\varphi\left[r+{ }_{\frac{r}{r}} \ln \left(\frac{q}{r}\right)\right\rfloor \\
& *\left[\frac{\partial r}{\partial \theta}+\frac{1}{q r} \frac{\partial q}{\partial \theta}-\frac{1}{r^{2}} \partial \theta\left(\ln \left(\frac{q}{r}\right)+1\right)\right] \\
& \frac{\partial q}{\partial \theta}=\frac{4}{\left(\gamma_{G} \hat{\theta}-2\right)^{2}} \\
& \frac{\partial r}{\partial \theta}=\left\{\begin{array}{c}
\operatorname{sign}(\hat{\theta})\left\{2\left[\hat{\theta}\left(-\beta_{2}\right)-K_{Z s}(\hat{\theta})\right]\right\}^{-1 / 2} \\
*\left[-\beta_{2}+2 / \gamma_{G}+4 /\left(2 \gamma_{G}-\gamma_{G}^{2}\right)\right]\left(\gamma_{G} \neq 0\right) \\
\operatorname{sign}(\hat{\theta})\left\{2\left[\hat{\theta}\left(-\beta_{2}\right)-K_{Z s}(\hat{\theta})\right]\right\}^{-1 / 2} \\
\quad *\left(-2 \beta_{2}-\hat{\theta}\right)\left(\gamma_{G}=0\right)
\end{array}\right. \\
& \frac{\partial \theta}{\partial \beta_{2}}=-\frac{4}{\left(\gamma_{G} \beta_{2}-2\right)^{2}} \\
& \frac{\partial \beta_{2}}{\partial \mu_{G}}=\frac{1}{\sigma_{G}} \\
& \frac{\partial \mu_{G}}{\partial \boldsymbol{X}^{\mathrm{T}}}=\left\lfloor\frac{\partial G}{\partial X_{1}}, \frac{\partial G}{\partial X_{2}}, \cdots, \frac{\partial G}{\partial X_{n}}\right\rfloor \\
& \left.\frac{\partial P_{f}}{\partial \beta_{2}}=\varphi\left\lfloor r+\frac{1}{r} \ln \left(\frac{q}{r}\right)\right\rfloor 1-\frac{1}{r^{2}}\left(\ln \left(\frac{q}{r}\right)+1\right)\right\rfloor \frac{\partial r}{\partial \beta_{2}} \\
& \frac{\partial r}{\partial \beta_{2}}=-\operatorname{sign}(\hat{\theta})\left\{2\left[\hat{\theta}\left(-\beta_{2}\right)-K_{\text {Zs }}(\hat{\theta})\right]\right\}^{-1 / 2} \hat{\theta} \\
& \frac{\partial \beta_{2}}{\partial \sigma_{G}}=-\frac{\mu_{G}}{\sigma_{G}^{2}} \\
& \frac{\partial \sigma_{G}}{\partial \operatorname{Var}(\boldsymbol{X})}=\frac{1}{2 \sigma_{G}}\left\lfloor\frac{\partial G}{\partial \boldsymbol{X}} \otimes \frac{\partial G}{\partial \boldsymbol{X}}\right\rfloor
\end{aligned}
$$

where $\varphi(\cdot)$ is the probability density function of a standard normal distribution.

The formulas developed in this section is novel and can be used straightforwardly when the statistical responds of the structure is obtained using the statistical perturbation method.

\section{Illustrative examples}

\subsection{A shaft in a speed reducer}

The performance function of a shaft in a speed reducer is defined as ${ }^{[1,5]}$

$$
g(\boldsymbol{X})=S-\frac{32}{\pi D^{3}} \sqrt{\frac{F^{2} L^{2}}{16}+T^{2}}
$$

where $\boldsymbol{X}=[S, D, F, L, T]^{\mathrm{T}}$ is the random variable vector, and the distribution details are given in Table I.

\begin{tabular}{llll}
\hline Variable & Distribution & Parameter 1 & Parameter 2 \\
\hline Strength $S$ & Uniform & 50 & 60 \\
Diameter $D$ & Normal & 39 & 0.1 \\
External force & Gumbel & 1500 & 150 \\
$F$ & Normal & 400 & 0.1 \\
Span $L$ & Normal & 250 & 35 \\
Torque $T$ &
\end{tabular}

Table I: Distribution details of random variables in example

*For a uniform distribution, Parameter 1 and 2 are lower and upper bounds, respectively. For a normal and gumbel distribution, Parameter 1 and 2 are mean and the standard variance, respectively.

5.1

According to the third moment saddlepoint approximation, the failure probability of the speed reducer under random inputs could be obtained as

$$
P_{f}=6.6230 \mathrm{e}-04
$$

The reliability sensitivity with respect to the mean and the variance of the input random variables are then calculated with the developed formulas, which are

$$
\begin{gathered}
\frac{\partial P_{f}}{\partial \boldsymbol{X}^{\mathrm{T}}}=\left[\begin{array}{l}
\partial P_{f} / \partial S \\
\partial P_{f} / \partial D \\
\partial P_{f} / \partial F \\
\partial P_{f} / \partial L \\
\partial P_{f} / \partial T
\end{array}\right]^{\mathrm{T}}=\left[\begin{array}{l}
+1.1442 \mathrm{e} 5 \\
+2.2670 \mathrm{e} 5 \\
-1.9647 \mathrm{e} 3 \\
-7.3678 \mathrm{e} 3 \\
-0.0327
\end{array}\right]^{\mathrm{T}} \\
\frac{\partial P_{f}}{\partial \operatorname{Var}(\boldsymbol{X})}=\left[\begin{array}{l}
\partial P_{f} / \partial \operatorname{Var}(S) \\
\partial P_{f} / \partial \operatorname{Var}(D) \\
\partial P_{f} / \partial \operatorname{Var}(F) \\
\partial P_{f} / \partial \operatorname{Var}(L) \\
\partial P_{f} / \partial \operatorname{Var}(T)
\end{array}\right]^{\mathrm{T}}=\left[\begin{array}{l}
+6.5573 \mathrm{e} 3 \\
+2.5742 \mathrm{e} 4 \\
+1.9335 \\
+2.7189 \mathrm{e} 1 \\
+5.3708 \mathrm{e}-10
\end{array}\right]^{\mathrm{T}}
\end{gathered}
$$

The reliability sensitivity with respect to the mean of the random variables shows that the failure probability of the speed reducer increases as $S$ and $D$ increase, but descends as $F, L$ and $T$ rises. Torque $T$ has the highest impact on the model output, then is external force $F$ and span $L$, strength $S$ and diameter $D$ has a relatively smaller effect on the structural performance.

\subsection{A one-story one-bay frame}

As shown in Figure 1 is a one-story one-bay frame and the four potential failure modes of the system can be readily identified and defined by four linear limit state functions. The probabilistic properties of random variables are listed in Table II. 


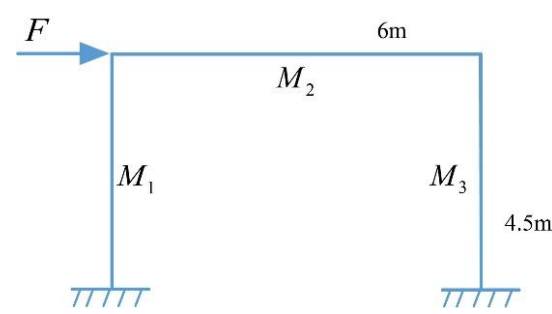

Figure 1: A one-story one-bay frame

\begin{tabular}{llll}
\hline Variable & Distribution & Mean & Std. \\
\hline$M_{1}$ & Lognormal & 200 & 40 \\
$M_{2}$ & Lognormal & 200 & 40 \\
$M_{3}$ & Lognormal & 200 & 40 \\
$F$ & Lognormal & 90 & 20 \\
\hline
\end{tabular}

Table II: Distribution details of random variables in example 5.2 .

The four potential failure modes of the one-story one-bay frame are listed as follows,

$$
\begin{gathered}
g_{1}=2 M_{1}+2 M_{3}-4.5 F \\
g_{2}=2 M_{1}+M_{2}+M_{3}-4.5 F \\
g_{3}=M_{1}+M_{2}+2 M_{3}-4.5 F \\
g_{4}=M_{1}+2 M_{2}+M_{3}-4.5 F
\end{gathered}
$$

Since this is a series system, the limit state function $G$ of the structural system can be defined as

$$
G=\min \left[g_{1}, g_{2}, g_{3}, g_{4}\right]
$$

The reliability and reliability sensitivity results can be obtained with the presented reliability sensitivity method. Thus, the system reliability could be calculated as

$$
P_{f s}=9.2617 \mathrm{e}-04
$$

The reliability sensitivity with respect to the mean and the variance of random variables are obtained as

$$
\begin{gathered}
\frac{\partial P_{f s}}{\partial \boldsymbol{X}^{\mathrm{T}}}=\left[\begin{array}{l}
\partial P_{f s} / \partial M_{1} \\
\partial P_{f s} / \partial M_{2} \\
\partial P_{f s} / \partial M_{3} \\
\partial P_{f s} / \partial S
\end{array}\right]^{\mathrm{T}}=\left[\begin{array}{l}
-2.3965 \mathrm{e}-4 \\
-2.3965 \mathrm{e}-4 \\
-4.7930 \mathrm{e}-4 \\
+0.0011
\end{array}\right]^{\mathrm{T}} \\
\frac{\partial P_{f s}}{\partial \operatorname{Var}(\boldsymbol{X})}=\left[\begin{array}{l}
\partial P_{f s} / \partial \operatorname{Var}\left(M_{1}\right) \\
\partial P_{f s} / \partial \operatorname{Var}\left(M_{2}\right) \\
\partial P_{f s} / \partial \operatorname{Var}\left(M_{3}\right) \\
\partial P_{f s} / \partial \operatorname{Var}(S)
\end{array}\right]^{\mathrm{T}}=\left[\begin{array}{l}
+2.9396 \mathrm{e}-6 \\
+2.9396 \mathrm{e}-6 \\
+1.1758 \mathrm{e}-5 \\
+5.9528 \mathrm{e}-5
\end{array}\right]^{\mathrm{T}}
\end{gathered}
$$

It can be clearly seen form the above matrix that the random $S$ with the value of +0.0011 has the greatest impact on the reliability and the increase of which would reduce the reliability level of the structural system.

\section{Conclusions}

A moment-based reliability sensitivity method is proposed in this paper. By combining the statistical perturbation method and the TMSA, the probability of failure is efficiently and accurately evaluated. The reliability-based parameter sensitivity analysis is then performed straightforwardly according to the sensitivity concept, and the formulas of the reliability sensitivity is derived. Two numerical examples verify the approach is effective in the reliability sensitivity estimation.

\section{Acknowledgements}

This work was supported by National Natural Science Foundation of China (Grant No. 51405490), Program for Changjiang Scholars and Innovative Research Team in University (Grant No. IRT_16R68), and National key research and development program (Grant No. 2016YFC0600905).

\section{References}

[1] Du, X., Sudjianto, A. First order saddlepoint approximation for reliability analysis. AIAA journal, 42(6): 1199-1207, 2004.

[2] Echard B, Gayton N, Lemaire M. AK-MCS: an active learning reliability method combining Kriging and Monte Carlo simulation. Structural Safety, 33(2): 145154, 2011.

[3] Grigoriu M. Methods for approximate reliability analysis. Structural Safety, 1(2): 155-165, 1983.

[4] Guo, S X. An efficient third-moment saddlepoint approximation for probabilistic uncertainty analysis and reliability evaluation of structures. Applied Mathematical Modelling, 38(1): 221-232, 2014.

[5] Huang, B., Du, X. Probabilistic uncertainty analysis by mean-value first order saddlepoint approximation. Reliability Engineering \& System Safety, 93(2): 325-336, 2008.

[6] Hurtado, J. E. Filtered importance sampling with support vector margin: a powerful method for structural reliability analysis, Structural Safety, 29(1): 2-15, 2007.

[7] Lu H, Zhang Y. Reliability-based robust design for structural system with multiple failure modes. Mechanics Based Design of Structures and Machines, 39(4): 420-440, 2011.

[8] Lu Z, Song S, Yue Z, et al. Reliability sensitivity method by line sampling. Structural Safety, 30(6): 517-532, 2008

[9] M. Tichy. First-order third-moment reliability method, Structural Safety, 16(3): 189-200, 1994.

[10] Rackwitz, R. Reliability analysis - a review and some perspectives, Structural Safety, 23(4): 365-395, 2001.

[11] Wu Y T. Computational methods for efficient structural reliability and reliability sensitivity analysis. AIAA Journal, 32(8): 1717-1723, 1994.

[12] Zhao, Y. G., Ang, A. H. System reliability assessment by method of moments. Journal of Structural Engineering, 129(10): 1341-1349, 2003. 